\title{
¿Cuál es la importancia de implementar estrategias en las organizaciones?
}

\author{
Clara Carmona de Ríos ${ }^{1}$
}

\section{Resumen}

Las estrategias que desarrollan las organizaciones son un pilar fundamental para saber la forma en que éstas van a competir en los mercados y de ellas dependerá el éxito de la gestión empresarial. Es de gran importancia que las organizaciones puedan identificar las estrategias más eficaces para competir eficientemente en los mercados. Identifcando e implementando la estrategia y bajo el enfoque de dirección estrategica, la cual es considerada un cumulo de enfoques u opciones (Hernández, Cardona, \& Del Rio, 2017) se pueden alcanzar las metas y objetivos de la organización. Este documento pretende hacer una aproximación de cinco tipologías de estrategias competitivas: Miles y Snow (1978), Porter (1980), Chandler (1971), Mintzberg (1988) y Kotler (1992). En este, se presentan sus cuestionamientos, similitudes y diferencias, asi como la importancia de implementar adecuadamente las estrategias en las organizaciones.

\section{Palabras Claves}

Organizaciones, Estrategias, Estrategias Competitivas, Diferenciación, Enfoque.

\begin{abstract}
The strategies developed by organizations are a fundamental pillar to know how they will compete in the markets and the success of business management will depend on them. It is of great importance that organizations can identify the most powerful strategies to compete efficiently in the markets. Only by establishing the right strategy can the goals and objectives of the organization be achieved. This article aims to analyze the five most important competitive strategies in the literature: Miles and Snow (1978), Porter (1980), Chandler (1971), Mintzberg (1988) and Kotler (1992). It is about finding their questions, similarities and differences and is intended to answer the importance of implementing appropriate strategies in organizations.
\end{abstract}

\section{Keywords}

Organizations, Strategies, Competitive Strategies, Differentiation, Approach.

\footnotetext{
1.Estudiante de Maestría en Administración, Universidad Autónoma de Baja California. claracarmona27@hotmail.com
} 


\section{Introducción}

Estrategia, es una de las palabras más usadas en el mundo de los negocios y las organizaciones, hoy en día es muy común que las grandes iniciativas tengan algo de estrategia, sin que se tenga mucha claridad de lo que implica, así como los desafíos de su implementación y el seguimiento que esta requiere para poder evidenciar los efectos de la misma.

El presente documento abordará la composición de los teóricos dando respuesta al origen de su tipología proporcionándole al lector elementos para poder identificar las características y bondades para ser identifica y aplicada de acuerdo al contexto en el que se encuentre la organización.

Inicialmente se presentan algunas de las definiciones usualmente utilizada por los gerentes en las organizaciones en diferentes latitudes:

Igor Ansoff, (1976) la define la estrategia como la dialéctica de la empresa con su entorno. El considera que la planeación y la dirección estratégica son conceptos diferentes, plantea la superioridad del segundo.

Tabatorny y Jarniu (1975) expresa que es el conjunto de decisiones que determinan la coherencia de las iniciativas y reacciones de la empresa frente a su entorno.

Charles Hoffer \& Schendel, (1978) expresa que la estrategia esta centrada en identificar "las características básicas que tiene la organización, y que están en concordancia con las necesidades que plantea el entorno".

A partir de lo anterior plantearemos la estructura de algunas de las tipologías proporcionándole al lector aspectos relevantes para lo cual se han identificado a los siguientes autores: Miles y Snow (1978); Porter (1980); Chandler (1971); Mintzberg (1988) y Kotler (1992).

\section{Tipologías estratégicas}

Miles y Snow (1978) expresan una de las tipologías de estrategias de negocios más populares, y que está basada en tres grandes hipótesis: La primera de ellas es que las organizaciones con éxito desarrollan a lo largo del tiempo una adaptación sistemática al entorno, con esta premisa, se destaca el "ciclo adaptativo" como la representación de "una filosofía general de comportamiento organizativo" (Miles y Snow, 1978: 27). Este ciclo de adaptación refleja las distintas aproximaciones de las organizaciones para enfrentarse a tres tipos de problemas en su entorno competitivo: problemas de empresa, relacionados con la definición de las combinaciones producto-mercado en las que operan; problemas de ingeniería, centrados en el sistema técnico de la organización; y problemas administrativos, relativos a los asuntos de estructura y procesos (González, 2001).

La segunda premisa es que existen cuatro orientaciones estratégicas dentro de cada industria. El descubrimiento de estos patrones de comportamiento se realizó basándose en estudios de campo en cuatro industrias diferentes -electrónica, proceso de alimentos, salud y libros de texto-, identificando tres tipos recurrentes viables y un cuarto que no representaba un comportamiento estratégico coherente. Esta premisa ha sido probada posteriormente en múltiples estudios que han validado la existencia de estos tipos en diferentes sectores de actividad (Snow y Hambrick, 1980; Hambrick, 1983; Miller, 1986; Zajac y Shortell, 1989; Conant et al.,1990; Shortell y Zajac, 1990; Zahra, 1996 y Lado, 1997). Las diferencias principales entre estos tipos se encuentran en la proporción de cambio en el dominio organizativo. Así, establecieron los siguientes patrones: Defensores, analizadores, prospectores y reactores.

Las organizaciones defensivas poseen un dominio estrecho y controlan nichos seguros en sus industrias. En el extremo opuesto se encuentran las empresas prospectoras, éstas buscan constantemente nuevas oportunidades de mercado y de desarrollo de productos. Las empresas analizadoras muestran características de los dos tipos anteriores. El último comportamiento identificado, el reactivo, supone un patrón disfuncional, ya que las organizaciones no siguen una estrategia conscientemente y su rendimiento es inferior al de los tipos anteriores.

Anfibios ISSN: 2665-1513 
La tercera premisa de esta tipología se refiere al resultado obtenido con cada estrategia. De este modo, Miles y Snow (1978) afirman que si los comportamientos prospector, defensivo y analizador se implantan en la organización adecuadamente llevará a un resultado efectivo. Sin embargo, no todos los autores piensan lo mismo sobre esta premisa, ya que algunas investigaciones la avalan (Wright et al., 1991; Zahra y Pearce, 1990) y otras son contrarias a la teoría (Snow y Hrebiniak 1980). Por consiguiente, los resultados que obtengan dependerá en gran medida de la correcta implementación de éstas y de la coherencia entre los tres elementos que constituyen el ciclo adaptativo de la organización, de forma que cada uno de los patrones enfatizará en diferentes funciones para producir un conjunto de capacidades distintivas sostenibles.

La organización reactiva carece de consistencia entre los citados tres elementos y por tanto no se adaptará al entorno; en consecuencia, sus resultados serán muy bajos. Son varios los estudios empíricos que han analizado la validez de la tipología de Miles y Snow. Hambrick (1983), Miller (1986), y Snow y Hambrick (1980) encontraron que los tipos estratégicos varían según diferentes características funcionales -principalmente I+D de producto y esfuerzo de marketing-, y que las diferencias en la efectividad de las diferentes estrategias dependían del entorno. Zahra y Pearce (1990), sin embargo, pusieron de manifiesto las limitaciones de este análisis donde no se ha prestado atención a la validación de las tipologías, y no se ha proporcionado excesiva información acerca de los procedimientos de clasificación. De forma similar, Hooley et al. (1992) indican las limitaciones del modelo en estudios inter-sectoriales donde las tipologías pueden resultar demasiado amplias para distinguir entre todos los posibles grupos estratégicos.

Porter (1980), expresa la composición de estrategias genéricas: liderazgo global en costos, diferenciación y enfoque o concentración, a través de ellas una empresa puede hacer frente a las cinco fuerzas que moldean la competencia en un sector y conseguir una ventaja competitiva sostenible que le permita superar a las firmas rivales.

Ediciones EFIM

\section{Figura.1 Las tres estrategias genéricas de Porter.}

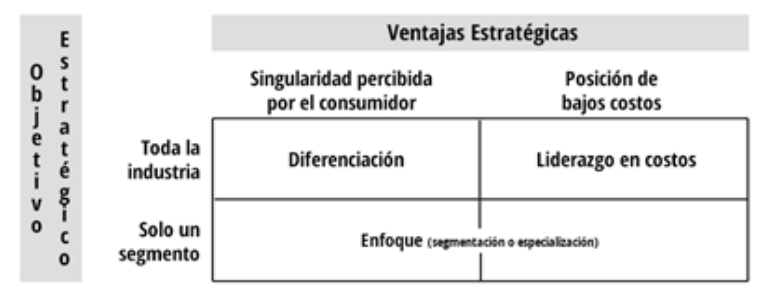

Fuente: Porter (1980, p. 81)

En la estrategia de liderazgo global en costos, la empresa debe tener la capacidad de reducir costos en todos los eslabones de su cadena de valor, de tal manera que la disminución de gastos redunde en un mejor precio para el consumidor y en consecuencia en una mayor participación de mercado.

En la estrategia de diferenciación la empresa debe producir servicios/productos exclusivos que sean percibidos así por los consumidores, quienes están en disposición de pagar más por tenerlos.

Con una estrategia de enfoque o concentración (segmentación o especialización) la empresa se concentra en satisfacer segmentos bien definidos, de población, de productos o geográficos.

Las dos primeras, liderazgo en costos y diferenciación, buscan la ventaja general en su sector, mientras que la tercera, enfoque, busca tener la ventaja dentro de un mercado objetivo.

Esta estrategia requiere un conocimiento detallado y profundo de las actividades de la cadena de valor para identificar aquellas en las cuales se pueden alcanzar ventajas en costos. El análisis de las actividades en la cadena de valor se realiza desde el punto de vista de los impulsores de costos. El principal motor en la estrategia de liderazgo de costos es la curva de experiencia, según la cual el costo unitario de producción disminuye con el número acumulado de unidades producidas. Esta ventaja se considera de carácter sostenible, puesto que no es fácil de imitar por los seguidores mientras no cambie la tecnología de producción. En contraste, las 
economías de escala pueden ser imitadas más fácilmente. (Francés, p.107)

La Diferenciación, Implica que la unidad de negocios ofrece algo único, inigualado por sus competidores, y que es valorado por sus compradores más allá del hecho de ofrecer simplemente un precio inferior. Es necesario comprender la fuente potencial central de diferenciación que surge de las actividades de la cadena de valor y el despliegue de la pericia necesaria para que dichas potencialidades se hagan realidad. La diferenciación exige la creación de algo que sea percibido como único en toda la industria. Los enfoques respecto de la diferenciación pueden adquirir muchas formas: diseño o imagen de marca, tecnología, características, servicio al cliente, red de corredores u otras dimensiones. (Hax y Majluf, p.163)

La estrategia de concentración, (tanto la basada en costes como en diferenciación) se caracteriza por la elección previa de un segmento, mercado local, fase del proceso productivo, etc. y por ajustar una estrategia óptima que responda a las necesidades específicas de los clientes escogidos. Se trata, en consecuencia, no de ser los mejores (en coste o en diferenciación) del mercado, pero sí de ser los mejores en el segmento escogido. La concentración puede apoyarse en la existencia de tipologías distintas de compradores (con estrategias de compra distintas), en la existencia de canales de distribución distintos (venta directa, por minoristas, por representantes, por correo, etc., etc.) y en la posibilidad de ofrecer variedades distintas de productos (tamaño, calidad, precio, prestaciones, etc.). (Larrea, p.98)

La tipología planteada por Mintzberg (1988) surge de la crítica que éste realiza a las estrategias genéricas de Porter, las cuales están más orientadas al análisis del entorno y contemplan escasamente el conjunto de recursos que son base para definir el dominio de mercado que se persigue. Mintzberg (1988) separó focalización de diferenciación y liderazgo en costes argumentando que la primera define el ámbito del mercado mientras que las otras dos estrategias de Porter reflejan la manera en que compite la empresa en ese mercado. Mintzberg también argumentó que tener un liderazgo en costes ba- sado en la estrategia de minimización de costes no proporciona una ventaja competitiva por sí misma. Por tanto, él señala que el liderazgo en costes viene a ser como una diferenciación en precio. En todo caso Mintzberg (1988) señala seis tipos de estrategias, atendiendo a la idea de que las empresas pueden tener una estrategia de indiferenciación o que la empresa puede diferenciarse de las otras empresas en imagen, calidad, diseño, precio y soporte.

La estrategia de indiferenciación se identifica en aquellas empresas que no tienen ningún factor sobre el que llevar a cabo la diferenciación o que copian intencionadamente a sus competidores.

\section{En la estrategia de diferenciación en imagen,} las empresas buscan la diferenciación mediante la publicidad tratando de alcanzar la lealtad de sus clientes. El objetivo es crear una percepción distintiva de los productos o servicios en la mente de los clientes.

La diferenciación en calidad se logra mediante altas prestaciones de sus productos. Requiere de fiabilidad, duración y un desempeño superior del producto en relación con el precio. - La diferenciación en diseño por su parte exige a la empresa un énfasis en $\mathrm{I}+\mathrm{D}$ de nuevos productos. La idea es perfeccionar las características del producto y del diseño a través de la investigación y desarrollo.

\section{La estrategia de diferenciación en precio es} viable sólo a través de la consecución de costes bajos, para luego poder ofrecer precios bajos.

En la diferenciación en soporte, las empresas crean un grupo de productos complementarios a los principales, con el objetivo de ofrecer una atención más amplia para atender las necesidades de sus clientes en el segmento.

De otra parte, Kotler (1992) señala que es necesario desarrollar una estrategia en base a evaluaciones realistas de la relación de fuerzas existentes y de definir los medios a poner en funcionamiento para alcanzar el objetivo fijado. Es así como establece cuatro tipos de estrategias competitivas según la participación de merca-

Anfibios ISSN: 2665-1513 
do: las estrategias del líder, del retador, del seguidor y del especialista.

La organización líder en un producto-mercado es aquella que ocupa la posición determinante y es reconocida como tal por sus competidores. El líder es a menudo un polo de referencia que las empresas competidoras se esfuerzan en atacar, imitar o evitar. La empresa líder es generalmente aquella que contribuye más directamente al desarrollo de mercado de referencia. La estrategia obviamente más natural que pone de relieve la responsabilidad del líder es la de desarrollar la demanda global, intentando descubrir nuevos usuarios del producto, de promover nuevos usos de los productos existentes, o también de aumentar las cantidades utilizadas por ocasión de consumo. Actuando así, la empresa líder amplía el mercado de referencia, lo que será, en definitiva, beneficioso para el conjunto de los competidores existentes. La empresa líder tiene un papel destacado en el mercado y contribuye especialmente al esfuerzo de marketing, mediante la mejora del producto y la extensión del mercado desarrollando nuevos canales de distribución y dirigiendo la política de precios. Este tipo de estrategia se observará, según Kotler, en las primeras fases de ciclo de vida de un producto-mercado, cuando la demanda global es ampliable y la tensión entre competidores es débil debido al elevado potencial de crecimiento de la demanda global. Para Kotler (1992), la vida de una empresa líder no es fácil a no ser que disfrute de un monopolio. Debe estar constantemente alerta ya que puede perder su puesto de privilegio en el camino y quedarse en una segunda o tercera posición. Estas empresas líderes son referencia para sus competidores que buscan atacar, evadir o imitar. Estas empresas quieren seguir siendo el número uno, lo cual requiere actuar en tres fuentes: primero la empresa debe encontrar formas de expandir la demanda total del mercado; segundo, debe proteger su actual cuota de mercado, mediante acciones defensivas y ofensivas; y tercero, puede intentar incrementar su cuota de mercado, incluso cuando el mismo se mantenga constante. La empresa líder no se debe conformar con su estado actual, sino que debe encabezar el sector en el desarrollo de ideas sobre nuevos productos, en el servicio a los consumidores, en la efectividad Ediciones EFIM en la distribución y en la reducción de costes, incrementando continuamente su competitividad y su valor para los consumidores. El líder siempre defenderá su posición, para lo cual puede utilizar diferentes estrategias: defensivas u ofensivas. Una estrategia defensiva tiene como objetivo reducir la probabilidad de ataque, diversificar los ataques a las áreas menos peligrosas y reducir su intensidad. Buscan proteger la cuota de mercado; sin embargo, el líder no puede defender todas sus posiciones en el mercado, por lo que deberá concentrar sus recursos donde más lo necesite. Esta estrategia es adoptada por empresas innovadoras, que una vez abierto el mercado, se ven atacadas por competidores imitadores.

La estrategia ofensiva, por su parte, se basa en el principio de que la mejor defensa es un buen ataque. Los líderes con mente ofensiva tratan de "dar el primer paso" para crear una ventaja competitiva duradera y una sólida reputación como líder. La clave para mantenerse a la ofensiva es una incesante búsqueda de la mejora y la innovación continuas. Luchar por convertirse en la fuente de nuevos productos, mejores características de rendimiento, mejoras en la calidad, mayores servicios al cliente y formas de reducir los costes de producción, no sólo ayuda a que el líder evite sentirse satisfecho, sino que también mantiene a los rivales a la defensiva, luchando por conservar el paso.

Las estrategias del retador, por su parte, son estrategias agresivas cuyo objetivo declarado es ocupar el lugar del líder. Esta estrategia es utilizada por las empresas que ocupan un segundo o tercer lugar en participación en el mercado. Para conseguir esto puede realizar estrategias agresivas, pero primero debe conocer los recursos y capacidades de que dispone en relación con la empresa líder para elegir la estrategia más adecuada. En este sentido, Kotler (1992) define varias posibilidades para atacar al líder: ataque frontal, ataque en flanco y ataque por rodeos. En el ataque frontal, la empresa debe tener más recursos que la empresa líder o fracasará. En este caso, la empresa lanza su ataque frontal tras concentrar todos sus recursos y ataca los puntos fuertes del oponente en lugar de los débiles. El ataque a flancos se utilizará cuando 
la empresa dispone de menos recursos que la líder y optará por atacar los puntos débiles de la empresa detectados en segmentos específicos o áreas geográficas. El ataque por rodeos implica lanzar una gran ofensiva a varios frentes a la vez, de forma que la empresa oponente deba proteger su frente, partes laterales y zonas más débiles al mismo tiempo. La empresa retadora deberá contar con recursos superiores y deberá ofrecer al mercado más de lo que ofrece el oponente de forma que su oferta sea indeclinable.

Kotler (1992) señala que básicamente son tres los tipos de empresa a las que puede atacar un retador:

Pueden atacar al líder del mercado. - Pueden atacar a otras empresas del mismo tamaño que no estén atendiendo bien al mercado y tengan problemas de financiación. - Pueden atacar a pequeñas empresas locales y regionales que no estén atendiendo al mercado y tengan problemas de financiación.

Con respecto a la estrategia del seguidor, Kotler (1992) señala que, en vez de atacar al líder, estas empresas persiguen un objetivo de coexistencia pacífica y de reparto consciente del mercado, alineado su actitud a la del líder reconocido del mercado. Es una estrategia que se desarrolla, a través de un comportamiento de adaptación al líder, por un competidor con una baja cuota de mercado. Es aquella estrategia que busca y se concentra en los segmentos en los que tiene una posición mayor a través de una mayor especialización con la que conseguir una mayor rentabilidad reduciendo la diversificación. En esta estrategia la mayoría de empresas no tratan de arrebatar clientela a la competencia, sino de presentar ofertas similares, normalmente copiando las del líder y dando lugar a gran estabilidad en las cuotas de mercado. Esto se puede resumir en tres importantes principios: comportamiento adaptativo al líder, coexistencia pacífica y no represalias del líder. Cuando las empresas son seguidoras e imitan al líder, no significa que estén desprovistos de estrategias ya que deben mantener el nivel actual de su clientela y tratar de incrementarlo poco a poco. Por lo tanto, deben mantener, en todo momento, bajos costes $\mathrm{y}$ alta calidad e incluso introducirse en nuevos mercados porque la imitación no implica necesariamente pasividad o ser una copia calcada del líder. En esta estrategia se pueden distinguir tres tipos de estrategias para imitadores:

Clónico: copia los productos del líder, la distribución y la publicidad. - Imitador: copia algunos aspectos del producto del líder, pero se diferencia de él en términos de envasado, políticas de precios y publicidad. - Imitador por adaptación: se basa en los productos del líder, los adapta e incluso a veces los mejora. Deben atender diferentes mercados para evitar problemas con el líder.

En la estrategia denominada como: Especialista, Kotler hace mención a que en la mayoría de las industrias existen empresas que se especializan en determinados nichos de mercado en lugar de atender a todos el mercado. Incluso se enfocan a segmentos amplios, buscando segmentos dentro de los segmentos, llamados nichos. Este fenómeno, la mayoría de veces, se da en pequeñas empresas con escasez de recursos, aunque las unidades de negocio de grandes empresas han seguido también esta estrategia. Lo principal es que empresas con pequeñas cuotas de mercado pueden llegar a ser altamente rentables a través de esta política de nichos. La ventaja del especialista procede de su capacidad para satisfacer de forma única las necesidades de ese segmento específico. Las características necesarias para que un nicho de mercado sea atractivo son:

Que sea suficientemente grande y con poder adquisitivo para ser rentable. - que tenga potencial de crecimiento.

El nicho debe ser poco atractivo para los competidores principales.

Los recursos y capacidades de la empresa deben ajustarse a las exigencias del segmento.

La empresa debe poder defenderse de los ataques que realicen sus competidores a través de la consolidación de las preferencias hacia sus productos.

Alfred Chandler realizó investigaciones profundas de las organizaciones norteamericanas en Anfibios ISSN: 2665-1513 
actividad en el periodo entre 1850 y 1920 , que fueron la base de la mayor parte de su trabajo posterior y que dieron origen a una nueva forma de ver la gestión perspectiva contingente.

Chandler fue el primer teórico en defender la creación de un plan estratégico antes de la elaboración de una estructura organizacional, o sea, la estrategia debe preceder a la estructura. Teorizó también el concepto de descentralización en las grandes empresas en auge de los años 60 y 70 defendiendo que la ventaja de las empresas multidivisionales era el hecho de que éstas permitían que los ejecutivos superiores dejasen de ser los únicos responsables por el destino de una empresa y pasaran a tener responsabilidades más rutinarias, ganando tiempo para dedicarse a otras tareas y pasando a asumir el compromiso de un planteamiento a largo plazo.

Defendió también la necesidad de coordinar el planteamiento estratégico de las sedes con las políticas de las unidades de negocio.

De sus estudios, realizados en cuatro grandes organizaciones norteamericanas (Du-Pont, General Motors, Standard Oil Co. y Sears Roebuck \& Co.), sobre la estrategia y la estructura organizacional, Alfred Chandler Jr. concluyó que "la estructura organizacional de las grandes empresas americanas fue siendo gradualmente determinada por su estrategia de mercado" y establece:

Acumulación de recursos y ampliación de capacidad productiva (final del siglo XIX): el rápido crecimiento urbano y la proliferación del ferrocarril un poco por todo el mundo llevaron al acentuado crecimiento de los sectores del hierro y del acero. En este periodo las empresas adoptaron estrategias de ampliación de la capacidad productiva a través de la acumulación de recursos y de integración vertical por medio de la adquisición de proveedores de materias primas, a fin de aprovechar mejor el beneficio de las economías de escala, y despreciando muchas veces el desarrollo de canales de distribución.

Racionalización del uso de recursos: la estrategia de crecimiento de la capacidad productiva y de integración vertical llevó a la acentuada Ediciones EFIM ampliación de la dimensión de las empresas y, consecuentemente, a la necesidad de organización de los recursos acumulados. Por otro lado, el exceso de capacidad productiva, notorio durante la gran depresión de los años 30 , originó la necesidad de contención de costes a través de la creación de estructuras funcionales con líneas de autoridad y de comunicación perfectamente definidas.

Continuación del crecimiento: la racionalización del uso de recursos productivos posibilitó una reducción de costes $\mathrm{y}$, consecuentemente, un aumento de la eficiencia. No obstante, la creciente saturación de los mercados y el gradual aumento de los niveles de competencias obligó a las empresas a diversificar sus negocios y a buscar nuevos productos y nuevos mercados. Una de las consecuencias directas de esta diversificación fue el surgimiento de departamentos de búsqueda y desarrollo y de ingeniería y diseño industrial.

Racionalización del uso de recursos en expansión: la creciente complejidad de productos y operaciones llevó a la creación de nuevas estructuras organizacionales, en particular la estructura divisional multidepartamentalizada. Esta nueva estructura organizacional consistía en la creación de divisiones autónomas e integradas, responsables por cada línea principal de productos. Esta forma de organización, soportada por la descentralización de las operaciones y centralización del control administrativo, obligó a las empresas a dar mayor importancia al planeamiento a largo plazo, en la definición de objetivos y en la evaluación del desarrollo de cada división.

Es así como los resultados de Alfred Chandler Jr. concluyen en que las alteraciones ambientales llevan a las empresas a adaptar sus estrategias y a alterar sus estructuras organizacionales de forma a permitir su ejecución. (Nunes, Paulo, 2017)

\section{Conclusiones y recomendaciones}

Después de revisar los diferentes postulados, se puede decir la importancia que tiene el hecho de 
que las organizaciones definan la estrategia que llevaran a cabo para competir en los mercados. Si se pretende lograr los objetivos y metas, la empresa debe tener muy claro cuál es su estrategia que va utilizar para dirigirse al mercado y a que segmento dirigirá su producto.

Muchos autores han propuesto diferentes tipos de estrategias buscando orientar a las organizaciones para el cumplimiento de los objetivos. Miles y Snow dan a conocer sus estrategias relacionando las organizaciones con la capacidad de adaptación al entorno y su orientación estratégica, así como el objetivo y metas que estas contemplen.

Porter por su parte, generó su clasificación atendiendo a la ventaja competitiva perseguida y al ámbito de consecución de la misma, teniendo tres puntos clave, Liderazgo de costos, enfoque, Diferenciación. Seguidamente, Miller trato de integrar a Miles y Snow y a Porter, considerando la utilización por parte de la empresa de cuatro dimensiones básicas. Mintzberg, habla de Dimensiones y las conciben desde la diferenciación, mientras que Chandler, habla de orden para las organizaciones, Estrategia-Estructura-Desempeño. Por último, Kotler distingue a las empresas en relación a la posición que tiene en el mercado y la actitud que adopta frente a otros competidores. Estas tipologías son de las más importantes tratadas en la literatura y tienen grandes similitudes entre sí, pero también tienen diferencias, discrepancias y cuestionamientos; sin embargo, todas tiene como objetivo guiar a las organizaciones, brindarles una orientación para competir y cumplir con sus metas en el mundo de los negocios.

En un mundo tan globalizado las organizaciones deben estar a la vanguardia de las nuevas tendencias, desafíos o retos que les exige el mercado que con el trae a consumidores más demandantes de productos innovadores, es por ello que las empresas deben estar en constante innovación y tener un paso delante de su competencia, es decir, tener una ventaja competitiva integra la cual contribuya al desarrollo óptimo de la actividad económica de la organización y el alcance de los objetivos a mediano y a largo plazo, todo esto es posible con la implementa- ción de estrategias relacionas con el producto y el comportamiento ante amenazadas de competidores. Por lo tanto, de manera personal la recomendación para una organización que desea perfeccionarse en ser mejor en lo que hace es que implemente estrategias propuestas por el señor Kotler, ya que este señor tiene muchas ideas y postulado de como penetrar en los mercados.

\section{Referencias bibliográficas}

Ansoff, h. (1976): "La estrategia de la empresa". Universidad de Navarra, Pamplona, Traducción del original: Corporate strategy, Mcgraw-hill, Nueva York, 1965

Ansoff, I. (1979): "The changing shape of the strategic problem, en SChENDEL, D. h.; hOfER, Ch. W". Strategic Management: a new view of business policy and planning, Little, brown and Company, pp. 30-44.

González, A. (2001): "Perfil competitivo y tecnológico de las empresas del sector de la electrónica: un análisis basado en la tipología de estrategias genéricas de Miles y Snow (1978)".

Documentos de Trabajo Universidad de Castilla La Mancha. Facultad de Ciencias Económicas y Empresariales, Serie 5, n.1, pp.1-59.

González, E. y ventura, J. (2007): “variedad estratégica y rentabilidad empresarial en la industria manufacturera". Revista de Economía Aplicada, n. 43, vol. xv, pp.7194.

Hernández, H. G., Cardona, D. A., \& Del Rio, J. L. (2017). Direccionamiento Estratégico: Proyección de la Innovación Tecnológica y Gestión Administrativa en las Pequeñas Empresas. Información tecnológica, 28(5), 15-22.

Kotler, P. (1992): "Dirección de Marketing". Ed. Prentice hall, Sétima edición, Madrid, España

Mintzberg, h. (1973): "Strategy making in three modes". California Management Review, vol.16, n.2, pp. 44-53.

Anfibios ISSN: 2665-1513 
Mintzberg, h. (1978): "Patterns in strategy formation". Management Science, vol. 24, n. 9, pp. 934-948.

Mintzberg, h. (1979): “An Emerging Strategy of Direct". Research, Administrative Science Quarterly, vol. 24, n. 4, pp. 582-589.

Mintzberg, h. (1988): "generic Strategies: Toward a Comprehensive framework". Advances in Strategic Management, n. 5, pp. 1-67. greenwich, CT: JAI Press.

Mintzberg, h. (1990). "Strategy formation: Schools of thought". (Ed.). Perspectives on Strategic Management. Nueva York, Harper Business: pp. 105-235.

Porter, M. (1985): "Estrategia Competitiva: Técnicas para el análisis de los sectores industriales y de la competencia. Editorial Continental, México Df.

Porter, M. (1990): "Ventaja Competitiva: Creación y sostenimiento de un desempeño superior". Editorial Secsa, 